\title{
8. Die Konferenz von Jalta und ihre Folgen
}

Die letzte Phase der Flucht der Rußlanddeutschen nach dem Westen ist nur in wenigen persönlichen Zeugnissen beschrieben ${ }^{1}$, das Ende des Fluchtwegs auf deutschem Boden liegt bis heute weitgehend im Dunkel. Die Erlebnisse waren auch hier chaotisch. Wer es geschafft hatte, unter unsäglichen Opfern, Strapazen und Verlusten das Gebiet des Altreichs zu erreichen, lebte nun in ständiger Angst, von den sowjetischen Suchkommandos aufgespürt und in die UdSSR abtransportiert zu werden. Mit der Repatriierung, dem letzten Abschnitt einer jahrelangen unfreiwilligen Migration, endete der Leidensweg der aus der Heimat Gerissenen in den Arbeitslagern hinter dem Ural. Die sowjetische Zwangsrepatriierung ist in jüngerer Zeit Gegenstand detaillierter Untersuchungen geworden ${ }^{2}$. Diese haben bezüglich des Schicksals mehr oder minder geschlossener Gruppen, wie etwa der Mitglieder der Wlasow-Armee, wohldokumentierte Ergebnisse gebracht. Doch läßt sich der Weg der ehemals sowjetischen Staatsbürger deutscher Nationalität in der Masse der weit über zwei Millionen Repatrianten nicht im einzelnen verfolgen und ihr Geschick mithin nicht isoliert erfassen. Angaben über Anzahl und geographische Lokalisierungen der Rußlanddeutschen in dieser dunkelsten und verworrensten Periode des Zweiten Weltkrieges beruhen ausschließlich auf Schätzungen und Mutmaßungen.

Man kann davon ausgehen, daß sich um die Jahreswende 1944/45 der größte Teil der Deutschen aus der besetzten Sowjetunion, etwa 200000 Personen, im Reichsgau Wartheland befand. Gauleiter Greiser hatte den ab Oktober 1944 in Panik geratenden Deutschen seines Gaus wiederholt versichert, daß die Weichsel-Linie von den Russen nicht gebrochen werden könne und für den Fall einer Verschlechterung der militärischen Lage alle Vorkehrungen für einen geordneten Abzug der Deutschen aus dem Warthegau getroffen seien ${ }^{3}$. Demgegenüber machten schon die Anweisungen

\footnotetext{
${ }^{1}$ Vgl. Schieder, Th., u. a. (Hrsg.), Dokumentation der Vertreibung der Deutschen aus Ost- und Mitteleuropa, Bonn (Bundesministerium für Vertriebene, Flüchtlinge und Geschädigte) 1953-1961, besonders Band I/1. In den letzten Jahren hat die Diskussion zu „Flucht und Vertreibung " in der Bundesrepublik Deutschland diese Fragen neu aufgeworfen. Ein Ergebnis der Diskussion ist auch die Dokumentation von Mühlfenzl, R. (Hrsg.), Geflohen und vertrieben. Augenzeugen berichten, Königstein/Taunus 1981; zu den Deutschen aus der UdSSR dort s.S.117-164. Allgemein ist das Schicksal der Deutschen aus der Sowjetunion in diesen Dokumentationen gegenüber dem der Deutschen aus Ostmitteleuropa stark unterrepräsentiert.

${ }^{2}$ Für die amerikanische Seite: Epstein, J., Die Zwangsrepatriierung von antikommunistischen Kriegsgefangenen in die Sowjetunion in: Politische Studien 22 (1971), Heft 196, S. 149-56; ders., Operation Keelhaul. The Story of Forced Repatriation from 1944 to the Present, Old Greenich Conn. 1973; der Nachlaß Epsteins, der die mühevolle Kleinarbeit seiner Ermittlungen zeigt, befindet sich im Archiv der Hoover Institution, Stanford/Ca. Für die britische Seite: Tolstoy, (Count) N., Die Verratenen von Yalta. Englands Schuld vor der Geschichte, München 1978.

${ }^{3}$ Shechtman, Population, S. $361 \mathrm{ff}$.
} 
des Reichsinnenministers (mit Gegenzeichnung Himmlers) vom 12. Oktober $1944^{4}$ deutlich, daß durchaus nicht vorgesehen war, die eingegliederten Ostgebiete überall vollständig von der deutschen Bevölkerung zu räumen - und wenn, dann erst in allerletzter Minute. Die Durchhalteparole sollte nach Möglichkeit auch auf dem zivilen Sektor befolgt werden.

$\mathrm{Zu}$ dieser Zeit befanden sich ca. 70000 Deutsche aus der UdSSR, die sog. A-Fälle, in den Beobachtungslagern sowie im Arbeitseinsatz im Altreich. Eine uns unbekannte Zahl von Aussiedlern und Flüchtlingen hielten sich noch in den Auffanglagern an den Grenzen des "Großdeutschen Reiches" sowie in anderen Vomi-Lagern im Generalgouvernement auf. Auffanglanger waren mit Eintreffen der Flüchtlingsströme aus dem Schwarzmeerraum entlang der östlichen und südöstlichen Reichsgrenze errichtet worden, in der Mehrzahl in requirierten Schlössern, Burgen oder Kirchenbesitz, so in Portsch im Salzburger Land ${ }^{5}$, in der Steiermark ${ }^{6}$ und in Bistritsa im Gebiet von Novi Sad ${ }^{7}$. In diesen Unterkünften werden in erster Linie Alte, Kranke und Sieche zurückgeblieben sein, als der Großteil der noch treckfähigen oder transportfähigen Familienmitglieder zur „Schleusung “ in die Lager von Litzmannstadt bzw. direkt zur "Ansetzung" in den Warthegau weiterzog. Im Lauf der Monate haben sich nun aus diesen Auffanglagern kleine Gruppen in Richtung Altreich abgesetzt, in erster Linie Personen, deren Familienangehörige oder Bekannte bereits als A-Fälle ins Altreich geschafft worden waren. Man darf davon ausgehen, daß die Zahl aller um die Jahreswende 1944/45 in den Auffang- und Schleusungslagern befindlichen Rußlanddeutschen kaum unter $25000 \mathrm{lag}$. Mindestens 20000 Männer mochten sich zu dieser Zeit bei den Einheiten der Wehrmacht und der Waffen-SS an der Front befinden; eine große Zahl aus den Reihen der Aussiedler war in anderen bewaffneten Formationen in der Umgebung ihrer Lagerbezirke oder Ansetzungsgebiete im Einsatz. Insgesamt befanden sich zwischen 335000 und 350000 aus der UdSSR ausgesiedelter Personen deutscher Herkunft oder Abstammung auf dem Boden des "Großdeutschen Reiches“ oder waren zur Verteidigung seiner Grenzen eingesetzt.

Anfang 1945 setzte die Rote Armee zum Blitzvormarsch an. Die entscheidende russische Großoffensive begann am 12.Januar. Nach dem Weichseldurchbruch erfolgten relativ schnell und unbehindert die Besetzung des Warthegaus, des Generalgouvernements und der Durchstoß zur Oder. Von dort trat die Rote Armee den Marsch auf Berlin an.

Evakuierungspläne waren für etwa 1,5 Millionen im Warthegau befindlicher Deutscher erstellt worden. In Anbetracht der sowjetischen Blitzoffensive erwiesen sie sich als völlig ungenügend. In vielen Kreisen des Warthegaus wuchs die Zahl der zu eva-

\footnotetext{
4 Der Reichsminister des Innern, II RV 4004/44-125 Wg, Berlin 1944, Betr.: Verhalten der Behörden bei Feindbesetzung, BA R 18/2876, fol. 1-31.

${ }^{5}$ Die Salzburger Zeitung vom 20.4. 1944 berichtete vom Eintreffen von 1000 deutschen Bauern aus dem Kaukasus, der Ukraine und dem Schwarzmeergebiet.

6 Der Ostdeutsche Beobachter vom 12. Juli 1944 berichtete von der Ankunft von 33000 deutschen Umsiedlern aus Rußland.

7 Deutsches Volksblatt vom 7. Juni 1944.
} 
kuierenden Personen über Nacht auf das Doppelte ${ }^{8}$. Himmlers Umsiedlungsmaschinerie hatte längst kapituliert; der Großteil der SS-Sonderkommandos hatte sich frühzeitig abgesetzt oder war anderweitig eingesetzt. Hoffmeyer fand auf dem übereilten Rückmarsch den Tod. An die Stelle einer irgendwie geordneten Rückzugsbewegung trat die individuelle Flucht. Doch ist, selbst nach offizieller Mitteilung deutscher Dienststellen in den letzten Kriegsmonaten, der Mehrheit der in den eingegliederten Ostgebieten wohnhaften oder vorübergehend untergebrachten Reichs- und Volksdeutschen die Flucht nicht mehr gelungen. Viele Hunderttausende von Deutschen, Angehörige der kämpfenden Truppen sowie reichs- und volksdeutsche Zivilpersonen, fielen in den eroberten polnischen Gebieten in die Hände der Roten Armee9. Unter diesen Personen waren Zehntausende Deutsche aus dem Schwarzmeer- und Wolgagebiet, aus dem Kaukasus und Donbas, aus den verschiedenen Teilen der Ukraine, aus Nord- und Zentralrußland. Viele von ihnen befanden sich nach monatelangen Trecks erneut auf einem Wintermarsch unter extrem harten Bedingungen. Sie erlebten dabei Diebstahl und Plünderung, Mord und Vergewaltigung, Krankheit und Tod. Wem immer es gelang, ins Altreich zu entkommen, erfuhr auch dort nur allzuoft einen totalen Mangel an Mitmenschlichkeit und Hilfsbereitschaft.

Die nationalsozialistischen Medien hatten die Nachricht verbreitet, daß alle Deutschen aus der UdSSR, die in die Hände der Alliierten gerieten, insbesondere die uniformtragenden Männer, an die Sowjetunion ausgeliefert würden. Dem schien der Text auf Millionen abgeworfener amerikanischer Flugblätter zu widersprechen: Die Aufforderung an die deutschen Soldaten zum Überlaufen war ausdrücklich mit dem Hinweis auf die Gültigkeit der Genfer Konvention versehen. 90\% aller in amerikanische Gefangenschaft geratener Deutscher trugen solche Flugblätter mit sich, hatten also die Hoffnung, nach den Grundsätzen der Genfer Konvention behandelt zu werden. Die deutsch-russischen Soldaten, die der Wehrmacht oder der Waffen-SS angehörten, versuchten dementsprechend, zu den westlichen Armeen überzulaufen. Viele vernichteten jedoch ihre deutschen Uniformen sowie alle Papiere, um nicht als Deutsche aus Rußland identifiziert werden zu können. Auch für deutsche Zivilpersonen, ehemals sowjetischer Staatsangehörigkeit war das Endziel das Innere Deutschlands, d.h. die von den westlichen Armeen eroberten Gebiete. Deutsche Familien aus der UdSSR, die sich in Deutschland aufhielten, versuchten, sich und ihre Identität zu verbergen.

$\mathrm{Zu}$ gegenseitigen Auslieferungen von Staatsangehörigen war es zwischen amerikanischen und sowjetischen Truppenkommandeuren bereits ab Juni 1944 gekommen, dem Zeitpunkt, an dem die Abteilung für psychologische Kriegführung der USArmee die großangelegte Flugblattaktion zum Überlaufen gestartet hatte. Mit der Annäherung der Fronten entwickelten sich die Auslieferungen zur Routineangelegenheit. Bei Nacht fuhren auf freiem Felde Militärlastwagen meist amerikanischer Herkunft mit Ladungen von Hunderten Kriegsgefangener ehemals russischer Staats-

\footnotetext{
${ }^{8}$ Neue Zürcher Zeitung vom 11.2. 1945.

9 Nachrichten- und Pressedienst, 7.2. 1945.
} 
angehörigkeit vor, die Offizieren der Sowjetarmee ausgehändigt wurden. Diese Praxis hatte viele Monate vor dem Abkommen von Jalta eingesetzt. In der ersten Septemberhälfte 1944 wurde ein britisch-sowjetisches Geheimabkommen geschlossen, in dem sich beide Seiten verpflichteten, Gefangene mit der Staatsangehörigkeit des Partners gegeneinander auszutauschen. Dieses Abkommen spezifizierte zum ersten Mal, daß die Auslieferung sowjetischer Staatsbürger ,unabhängig von dem Wunsche des Betreffenden, nach Rußland zurückzukehren oder nicht", stattfinden sollte: "Statements will not be taken from Soviet nationals in future as to their willingness to return to their native country. "10 Der Stab der Alliierten Mächte schloß sich dem sowjetisch-britischen Geheimabkommen an.

Die Maßnahmen, die mit dieser Zwangsauslieferung verbunden waren, standen in krassem Gegensatz zur Genfer Konvention wie auch zum Recht auf Asyl, das beispielsweise die USA traditionellerweise Flüchtlingen und Überläufern gewährten. In den Wintermonaten 1944/45 wurden auf Antrag der sowjetischen Botschaft aus den in den USA befindlichen Kriegsgefangenenlagern die sowjetischen Staatsangehörigen herausgesucht und unabhängig von ihrer Willensäußerung in die Sowjetunion geschickt. Doch Nachrichten von Selbstmorden und zahlreichen Selbstmordversuchen sickerten durch. George Kennan, damals Chargé d'Affaires der US-Botschaft in Moskau, gab einen ausführlichen Bericht darüber, wie die „Heimkehrer" im sowjetischen Hafen Murmansk mit Blasmusik begrüßt und dann unter schwerer Bewaffnung in unbekannte Richtung abgeführt wurden. Diese Informationen veranlaßten Joseph C. Grew, Unterstaatssekretär im State Department, in einer Note auf die Unvereinbarkeit solcher Maßnahmen mit den Grundsätzen des internationalen Rechts, insbesondere den Bestimmungen der Genfer Konvention, sowie des amerikanischen Asylrechtes aufmerksam zu machen. Doch seit Oktober 1944 hatte sich die offiziell als "neue Politik gegenüber sowjetischen Staatsbürgern" bezeichnete Leitlinie auch im State Department durchgesetzt. Grews Note blieb ungehört. Am 24. Oktober 1944 ließ die Sowjetregierung eine Pressenotiz erscheinen, derzufolge Generalhauptmann Filip Golikov zum „Delegierten für die Repatriierung sowjetischer Staatsbürger in Gefangenschaft oder Zwangsentfernung (von ihrer Heimat) in Deutschland und in den deutsch besetzten Gebieten" ernannt worden war ${ }^{11}$. Daß für seinen Stab höchste militärische Ränge vorgesehen waren, ließ erkennen, welche Bedeutung die Sowjetregierung der Repatriierung beimaß. Am 11. Februar 1945 schließlich unterzeichneten Vertreter der USA und der UdSSR in Jalta das Abkommen über den Austausch von Kriegsgefangenen und Zivilisten, die von der sowjetischen bzw. amerikanischen Armee befreit oder gefangengenommen worden waren. Die vorbereitenden Unterlagen für dieses Abkommen reichen bis auf den 17. Juni 1944 zurück. Der Wortlaut des Abkommens hingegen wurde erst nach Ablauf eines Jahres veröffentlicht ${ }^{12}$ - zu diesem Zeitpunkt war der Hauptteil der Repatriierung bereits abgeschlossen.

${ }^{10}$ Epstein, Operation, S. 29.

11 Vgl. Daily Telegraph vom 25. Oktober 1944.

12 United States Department of State, Executive Agreement Series 505. Publication 2530, Washington D.C. 1946. 
Artikel 1 des Abkommens von Jalta legte fest, daß alle sowjetischen Staatsbürger, die von den unter amerikanischem Oberbefehl operierenden Truppen, und alle Staatsbürger der USA, die von den unter sowjetischem Oberbefehl stehenden Heeresteilen befreit worden waren, unverzüglich von der Masse der feindlichen Kriegsgefangenen isoliert und bis zur Übergabe an den jeweiligen Partner, die an bestimmten Plätzen stattfinden sollte, getrennt gehalten werden mußten. Artikel 2 sprach den militärischen Vertretern der beiden Parteien wechselseitig das Recht auf unmittelbaren Zugang zu Lagern und Konzentrationspunkten, in denen sich ihre Staatsangehörigen befanden, zu, und autorisierte sie darüber hinaus, die innere Verwaltung, Disziplin und Leitung dieser Lager in Übereinstimmung mit den militärischen Verfahren und Gesetzen ihres Landes auszuüben. Der freie Abtransport ihrer Landsleute wurde gewährleistet. In Ausnahmefällen konnte ein solcher sogar ohne vorherige Informierung der verantwortlichen Stellen der anderen Partei erfolgen. Artikel 3 dehnte diese Bestimmungen auf Zivilpersonen aus. Die Sowjetführung erhielt damit das Recht, neben den fast drei Millionen Sowjetbürgern, welche die Rote Armee auf ihrem Vormarsch nach Westen in Gebieten Ostmittel- und Mitteleuropas überrollt hatte, mehr als zwei Millionen früherer Sowjetbürger zu repatriieren, die sich nun in den westlichen Besatzungszonen befanden oder zukünftig befinden würden. Unter ihnen war auch eine uns unbekannte Zahl ehemals sowjetischer Deutscher. Alle Personen, kriegsgefangene Soldaten und Offiziere, Zivilisten aller Art und displaced persons (DPs), die bis zum 1.September 1939 auf sowjetischem Territorium gelebt hatten und seit dem 22. Juni 1941 von dort entfernt worden waren oder sich entfernt hatten, unterlagen mit Inkrafttreten des Abkommens am 11. Februar 1945 der Repatriierung.

Das Abkommen von Jalta kam den sowjetischen Wünschen weitestgehend entgegen. In den nachfolgenden Verhandlungen stellte sich, veranlaßt durch immer lauter werdenden internationalen Protest, eine mildernde Nuance bezüglich der Behandlung der Repatrianten ein. Sie betraf u. a. Polen, Esten, Letten und Litauer, die nunmehr in ihre eigenen Staaten zurückkehren konnten. ${ }^{13}$ Russen aber, unter ihnen die 750000 Angehörigen der Wlasow-Armee und ihre Sympathisanten, Ukrainer, Kosaken, Kaukasier und alle anderen Völkerschaften der UdSSR, die in den Fremden Heeren Ost der deutschen Wehrmacht gegen das Sowjetregime gekämpft hatten, sollten restlos und erbarmungslos repatriiert werden. Dies bedeutete in vielen Fällen den Weg in den sicheren Tod, in den anderen den Weg in die Zwangs- und Arbeitslager auf zum Teil unbegrenzte Zeit. Dasselbe Schicksal traf die Deutschen aus der UdSSR. Ihr tragischer Weg zurück in die UdSSR war schon insofern vorgezeichnet, als sie nicht, wie etwa Polen und Ukrainer, Gruppen machtvoller Fürsprecher im westlichen Ausland besaßen, und sich keine der verhandelnden Nationen zu besonderer Nachsicht und Mildtätigkeit gegenüber einer nationalen Gruppe veranlaßt sah, die in hohem Maße der Kollaboration mit dem nazistischen Deutschland verdächtig war.

${ }^{13}$ Vgl. Frederikson, O.J., American Military Occupation of Germany, 1945-1953, Darmstadt (US Headquarters, Historical Division) 1953, S.76. 
Eine Ausnahme bildeten etwa 10000 Mennoniten meist holländischer Herkunft, die im Strom der Rußlanddeutschen die westlichen Besatzungszonen erreicht hatten. Sie wurden in den Repatriierungslagern bzw. in ihren Verstecken von Vertretern der nordamerikanischen Mennonitenverbände aufgesucht oder kontaktiert und über die geeignete Verhaltensweise unterrichtet bzw. mit entsprechenden Papieren versorgt. Auf diese Weise konnten sie dem Zugriff der sowjetischen Repatriierungsoffiziere entgehen. Sie wurden ab 1948 durch die International Refugee Organisation (IRO) nach Kanada und Paraguay verschifft ${ }^{14}$.

Die Massenrepatriierung sowjetischer Bürger fiel in die Zeit vom 20. Mai bis 30. September 1945. In diesen vier Monaten wurden allein aus den drei westlichen Besatzungszonen 2034000 betroffene Personen mit oder gegen ihren Willen, teils unter Anwendung von Gewalt (einschließlich massiver Waffengewalt) in die UdSSR zurückbefördert ${ }^{15}$. Von Ende September an wurden - aufgrund vorausgegangener Revolten in den Lagern, massenweiser Selbstmorde und der Proteste und verstärkten Öffentlichkeitsarbeit in den westlichen Ländern - alle Zivilisten ehemals sowjetischer Staatsangehörigkeit von der Zwangsrepatriierung ausgenommen. Sowjetische Bürger, die in deutscher Uniform angetroffen worden waren und gegen die nicht der zwingende Verdacht bestand, daß sie als Kriegsverbrecher im Westen abzuurteilen seien, wurden weiter ausgeliefert. Erst im Herbst 1946 kam die Repatriierung zum Stillstand.

Um diese Zeit war die überwiegende Mehrheit der Rußlanddeutschen, die durch Flucht oder auf dem Wege der Administrativumsiedlung das besetzte sowjetische Territorium verlassen hatten, in die UdSSR zurückgeschafft. Die meisten der Geflohenen oder Ausgesiedelten, ca. 200000 Personen, waren 'bereits in den „eingegliederten Ostgebieten" vom Vormarsch der sowjetischen Truppen überrascht worden ${ }^{16}$. Nicht wenige wurden an Ort und Stelle liquidiert, die anderen in Eisenbahnwaggons unter unsäglich mühevollen Bedingungen in die Sammellager jenseits des Ural gebracht. Nach Berichten und Schätzungen sollen die Verlustquoten bei diesen Transporten z.T. zwischen 15 und 30\% gelegen haben. In Sibirien und Mittelasien erwartete sie die Einlieferung in die Sondersiedlungen (special'nye poselenija) für sowjetische Deutsche, wiederum stacheldrahtumzäunte Arbeitslager, die einem besonders scharfen Kontroll- und Überwachungssystem des NKWD unterstanden.

Etwa 70-80000 der Deutschen, welche die westlichen Besatzungszonen erreichen konnten, ist es nach Schätzungen der Landsmannschaft der Deutschen aus Rußland ${ }^{17}$ gelungen, der sowjetischen $Z$ wangsrepatriierung zu entgehen. Wenn man von etwa 350000 aus der UdSSR ausgesiedelten oder geflohenen Deutschen ausgeht, so käme man nach Abzug der obengenannten Zahlen, d.h. der ca. 200000 von der Roten Ar-

14 Barton, B., The Problem of 12 Million German Refugees in Today's Germany, Philadelphia (American Friends Service Committee) 1949.

15 Proudfoot, M.J., European Refugees 1939-1952. A Study in Forced Population Movement, London 1957, S. 211.

16 Schoenberg, Germans, S. 19.

${ }^{17}$ Persönliche Mitteilung von Dr. J.Schnurr, Stuttgart. 
mee Überraschten und der 70-80000 in den Westzonen der Repatriierung Entgangenen zu dem Schluß, daß sich unter den zwangsweise repatriierten ehemaligen Sowjetbürgern 70-80000 Personen deutscher Nationalität befanden. Ihr Schicksal war wie das der vor ihnen Deportierten die Einweisung in die Sondersiedlungen in Sibirien, Kasachstan und dem Altajgebiet.

In diesen Sondersiedlungen erreichten die Leiden der aus ihrer ursprünglichen Heimat gerissenen und dann so vielfach hin- und hergetriebenen Rußlanddeutschen ihren letzten Tiefpunkt. Die Isolation von der sowjetischen Bevölkerung und erst recht von der Weltöffentlichkeit war total. Erst in der Folge des Besuchs von Bundeskanzler Konrad Adenauer in Moskau setzte ihre partielle Rehabilitierung und damit die Milderung ihres außergewöhnlich harten Geschicks ein. 